\title{
Research on fusion method of elements in product design
}

\author{
Li Lin Yang
}

\author{
Zhengzhou University Of Industrlal Technology, Zhengzhou 450000, China \\ 349161801@qq.com
}

\begin{abstract}
This paper studies the performance method of modeling elements in product design, through the analysis of elements in the form of evolution form, explores the creative method of modeling elements, the visual expression of other elements and the visualization of the elements of the decomposition and product characteristics of graphic language through decomposition, formed with the design of both the intersection point after the new graphics are studied. Finally, using the new fusion method, the "bell" is used as the basic design element to design the appearance of the reducer.
\end{abstract}

Key words: product modeling, element, visualization, reducer

\section{Introduction}

Compared with the technological innovation, the appearance of innovative design is a kind of disruptive technology, is a low-cost innovation, is the core means of product innovation in the homogenization of competition.Thus, the product design is in the homogenization of competition is to develop international market, innovation design is of great significance, in product design, integration of elements and the appearance of a meeting point, for example, many designers are calling for China traditional culture and product integration, but it is difficult to find a a reasonable fit, the fusion methods of new elements become very important.

\section{Visual decomposition of elements}

The traditional domestic product design is relatively extensive type, only to consider the function of the product design, quality, use of the term, and so on, do not study some additional value, brand value. The integration of cultural elements will have a great improvement on the added value of products. For example, the application of traditional Chinese ink painting techniques in product design is not only a deduction of Chinese traditional art techniques, but also a taste and flavor of Chinese ink culture itself. With the help of three kinds of techniques of abstract, figurative and intention, we can reconstruct the visual elements, which will make the modern art of design in the context of globalization.

The visual element refers to the elements in the visual or visible forms, such as Chinese in traditional culture has many elements is invisible, concept and complexity, in order to make the elements can be more convenient to use in the product, some complex, immaterial, concept of elements in visualization form. Elements of the product design in the form of application before the first element of the visual image display, visibility of the elements mainly include patterns, symbols, scenes, objects and so on, as shown in figure 1. Secondly, it is necessary to decompose the elements in order to find the combination of the product features (Figure 2).

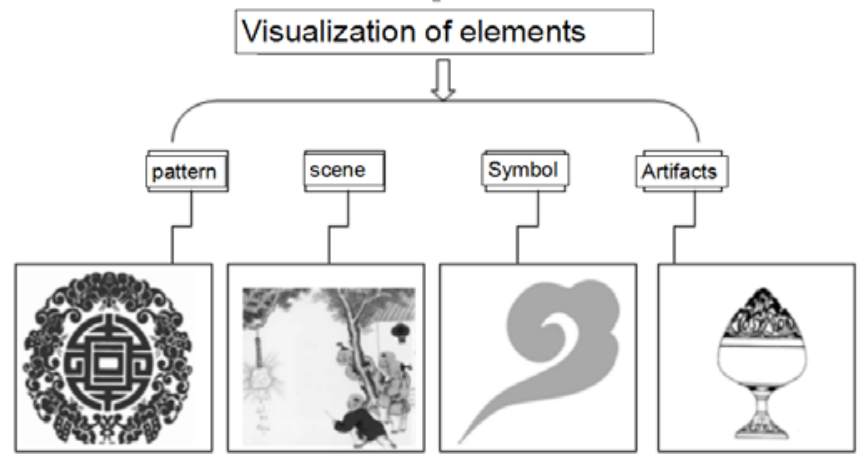

Fig. 1 Visibility element classification 


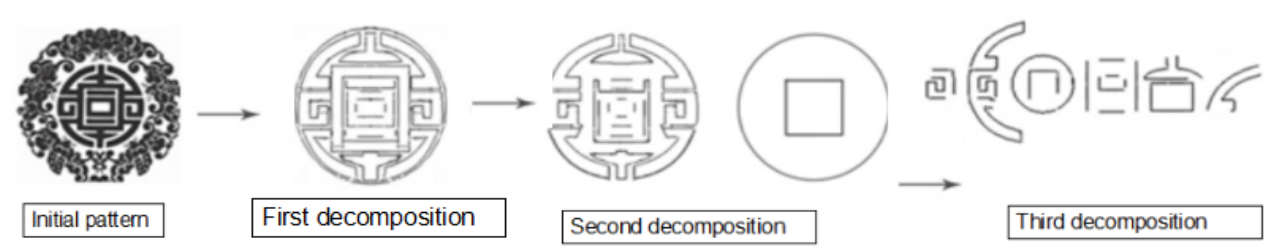

Fig. 2 Decomposition of visual elements

It is observed that a variety of objects outside the body, the first reaction will be caused by color, followed by shape. The most sensitive to visual stimulation, the fastest response is visual information symbols. Design elements content is rich and colorful, especially for the creation of personnel engaged in product design, need to know how to look beneath the surface of the problem, how to design the appropriate language expression, design elements into products, and then through the design language to the people, therefore, in the product design in the process of design elements will be changed from invisibility into visibility,It can be seen as visual awareness proposed in this paper.

\section{3. product of the characteristics of the language graphical decomposition}

product of the characteristics of the language graphical decomposition refers to the process of apart of the product for the decomposition of the process of refining the simplified graphics.The following will be a traditional Chinese elements into the design of reducer as an example to analyze.First of all, through the constraints in the design process of reducer,mainly from four aspects to the development of thinking(Figure 3),

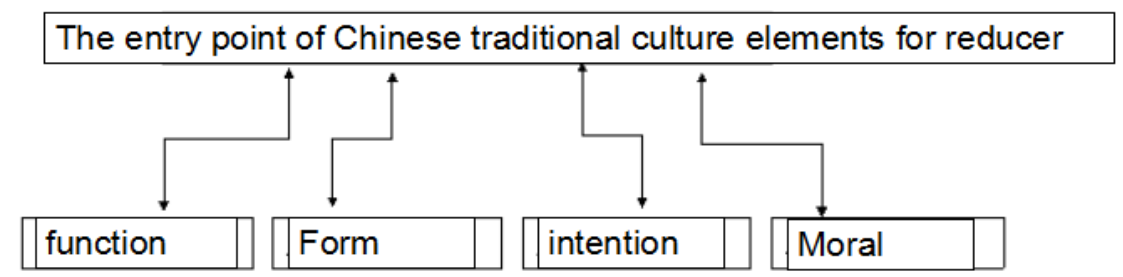

Figure 3 The entry point of traditional culture elements for reducer

The starting point for the function can be "strength and speed", these elements can be in a certain sense reflects the reducer and traditional culture under the influence of "vivid" and "perfect victory" "force", "quantity", "speed" of the traditional cultural elements in the form of search; the starting point is to "force", "square", "penetration", "stereo", these elements can give the product to a certain extent, the mighty impregnable feeling; from the reducer "speed", "change" and "calm" moral perspective to find out the traditional culture for China reducer elements of the "fortune", "Tang", "jade" and so on, from the reducer design intention to find the starting point of "mechanical" and "metal" and "Endurance", and then look for The elements of traditional culture fit, we can find that: "the spring and Autumn Period bronze", "Buddha", "clock", "Zen", "Terracotta Army" and so on, so these elements can express the feeling is "antique", "rich China flavor" and "auspicious" ancient oriental "the mysterious".

The basic characteristics of the reducer body is expressed by the surface or line, mostly based on the rectangle, can evolve different styles. The top line shows a narrowing trend, showing a steady upward trend; both sides of the waist line is a concave trend, showing elegant, beautiful style; both sides of the waist line is a convex trend, full of heavy, heavy feeling. At the same time, the irregular surface is rich and complex, and the rules are more concise and elegant. First, consider the main functional requirements of the box, the function of the subdivision and the principle of solving, to achieve a systematic program. Through the analysis and induction of the function of the box, the abstract function description is transformed into the concrete shape structure description.

It is the first step to choose the traditional Chinese cultural elements which is suitable for the reducer based on the traditional Chinese culture,after determining the elements of the Chinese traditional cultural elements of the cultural elements of visual decomposition, get the most simplified graphics to match the deceleration of the other (figure 4). Through the process of decomposition will dissolve 
into state graphic elements recently received original elements "point line surface", will China traditional cultural elements are all alternative decomposition and finishing standby.

\section{Fusion intersection formation}

From the various factors involved in the casting process of the reducer to find an entry point "form",this is mainly from the perspective of reducer morphological characteristics and the similarities between the material point of view, is in the overall shape of the "bell" spirit; it is not only a kind of tool use and China grade, ancient power and ancestral temple of the symbol, the craft is exquisite, appearance is one of the Chinese made solemn steady classic ancient bronze culture in

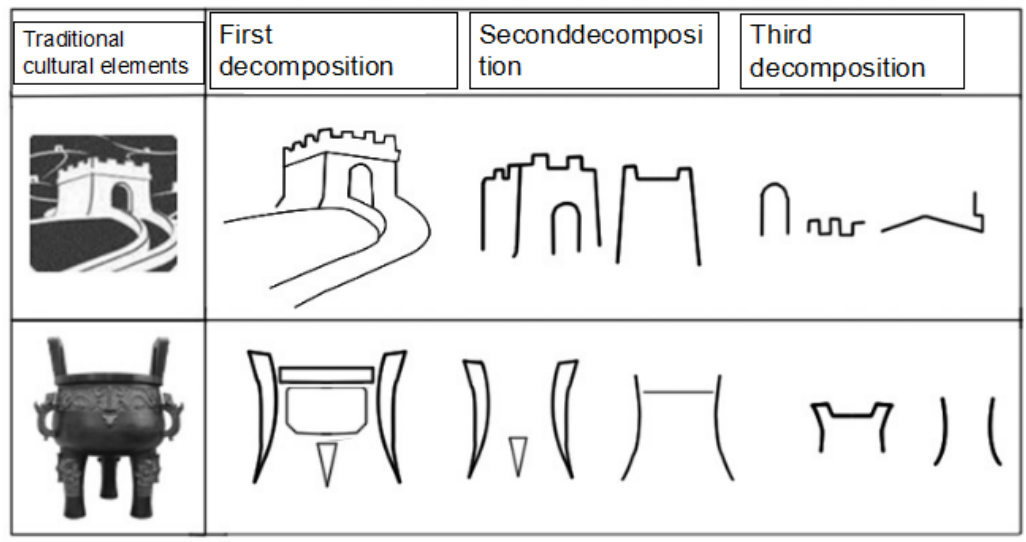

Figure 4 Alternative graphics after visual decomposition

manufacturing. "Embodied" clock appearance feeling from the meaning given is stable, stable, has a meaning town house, alert. Combined with the function of the reducer and the use of the environment will be integrated into the bell element of the overall shape, you can show a magnificent majestic momentum. In China traditional culture "bell" shape can be divided into many types, but each kind of form has a slight difference, here the similar part will bell shape to do visual decomposition such as (Figure 5), the visual elements in figure can be seen in the structure of the chain are the narrow width, from the point of the arrangement is to create a smooth sense of symmetry, sinking feeling.
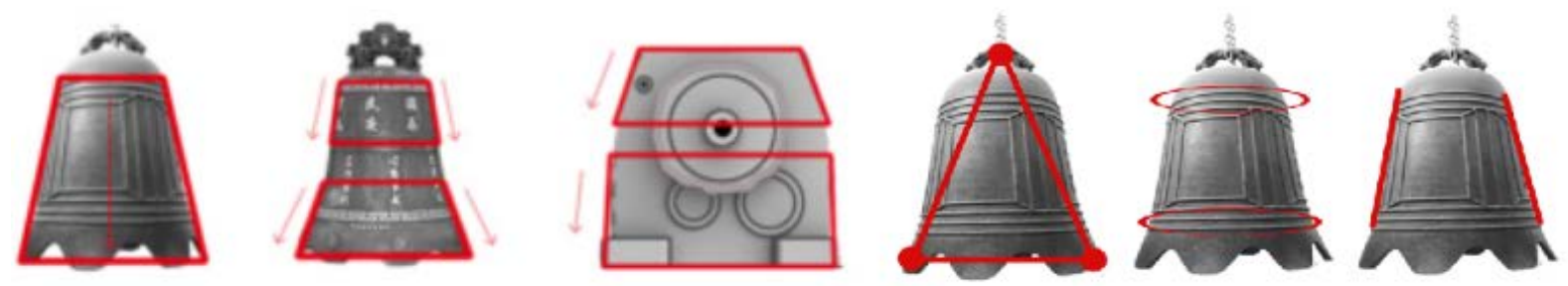

Figure 5 The extraction of the similarity of "bell" elements

Create a "bell" mainly from the element extraction and deformation, the extraction process requires full analysis of the "bell", element extraction requires a lot of deliberate experiments, the performance of different combination of abstract attempts to show the internal structure of the reducer of the overall shape of the building, the main structure for the completion of the final form of effect. Extraction of the evolution of the various forms of the clock (Figure 6), through the abstract deformation of the best simplified elements. The traditional layout method of "point, line and plane" to create a "clock potential", the design of the "potential" refers to "the characteristics and connotation of the clock itself; from the" reducer "appearance can be directly or indirectly feel the" clock elements "of the atmosphere.

\section{Conclusions}

In the form of shape, $\mathrm{R}$ series reducer main view, side view and top view of the main characteristics of the line, the feature point and feature surface as the improved object, without affecting the internal 
structure of the conditions of its shape which involved in the feature point and feature line, surface characteristics to adjust. The China elements of traditional culture "clock" for graphic decomposition, compare the features of language refined graphics and simple graphic reducer, select the most simple composed of point symbols, then looking for the intersection, finally determine the new graphic elements as the feature of language to facilitate the complex traditional Chinese cultural elements into the reducer "point, line and plane", finally obtains the intention form.

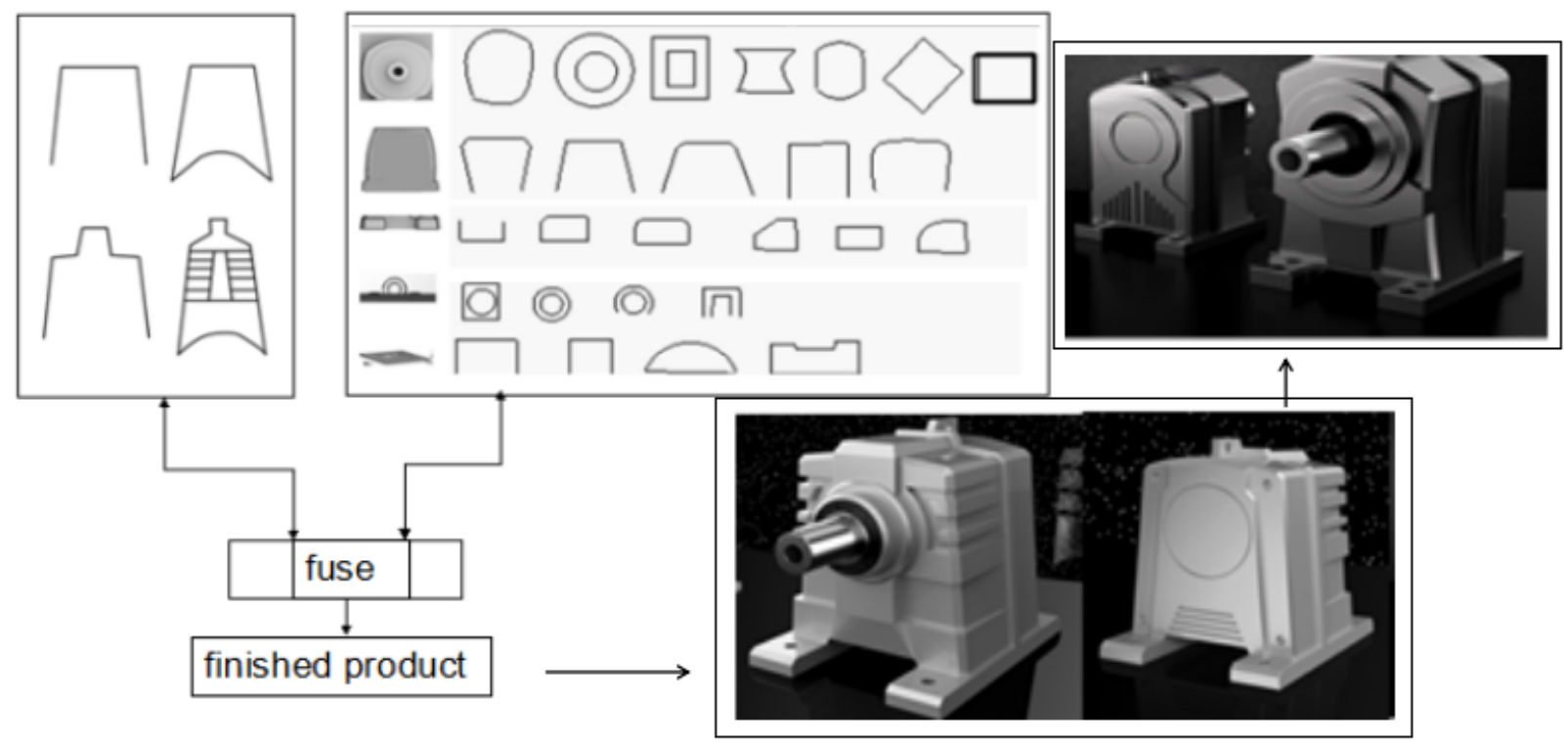

Figure 6 Forming of reducer with Bell element

In this paper, the selection of the traditional culture elements in the design of the reducer is explored. In the design process, the use of the other in the most basic "point", "line" and "surface" as the design foundation; combination of traditional culture Chinese unique recognition, visual beauty, meaning beauty, micro adjustment according to the aesthetic characteristics of the new era of abstract deformation on the traditional elements of fine, created from the recognition and the basic structure of the traditional reducer visual beauty combination, based on traditional culture form the basis for the elaboration of the modern reducer; put forward China traditional culture elements and other characteristics of reducer by the visual and graphic language based on the decomposition, thus creating a new intersection point, the formation of a new pattern to the ingenious combination; and to grasp the Chinese reducer of the elements of traditional culture Chinese taste: "momentum", "artistic conception".

\section{References}

[1].Erika MarsillacJames Jungbae Roh.Connecting product design, process and supply chain decisions to strengthen global supply chain capabilities [N]. // , 《Internation a l journal of production economics》2014 Jan. Pt.B

[2]Richie MoalosiVesna PopovicAnne Hickling-Hudson .Culture-orientated product design [C]. // , 《International journal of technology and design education》2010:2 .

[3]S. DengR. AydinC. K. KwongYun Huang.Integrated product line design and supplier selection: A multi-objective optimization paradigm [J]. // , 《Computers \&amp; Industrial Engineering》, 2014. [4]Qipeng Liao.On Modern Landscape Design Integrating Chinese Traditional Spiritual Culture[C]. // ,International Conference on Technology of Architecture and Structure (ICTAS)4th. [5]Ana ValenciaOscar PersonDirk Snelders.An in-depth case study on the role of industrial design in a business-to-business company [J]. // , 《Journal of Engineering and Technology Management 》,2013 